\title{
ON THE EXACT CALCULATION OF THE SCATTERING LENGTHS FOR LONG RANGE POTENTIALS. II. LENZ POTENTIALS
}

\author{
R. SZMYTKowski \\ University of Gdańsk, Institute of Theoretical Physics and Astrophysics \\ Wita Stwosza 57, 80-952 Gdańsk, Poland \\ (Received August 17, 1990)
}

The potentials vanishing asymptotically as Lenz potentials are considered and an exact method of calculating of the scattering lengths for them is presented. This method is especially useful for Buckingham polarization potential. Formulae obtained in this report are the generalization of those derived in the previous paper for the inverse power potentials.

PACS numbers: 03.80.+r, 34.10.+x

\section{Introduction}

Although the scattering length can be found numerically for any given interaction, analy tic formulae remain of considerable interest, especially for. understanding the dependences on the parameters characterizing potentials. In the previous paper [1] (to be referred to as I) we presented a method of the calculation of the scattering lengths for potentials for which the radial Schrödinger equation at $E=0$ can be solved analytically. We showed that the scattering length $a_{L}$ can be found as an asymptotic limit of the function $a_{L}(r)$ defined as:

$$
a_{L}(r)=r^{2 L+1} \frac{\mathcal{L}_{L}(r) r-(L+1)}{\mathcal{L}_{L}(r) r+L},
$$

where $\mathcal{L}_{L}(r)$ denotes the logarithmic derivative of a solution of the radial Schrödinger equation at $E=0$ :

$$
\mathcal{L}_{L}(r)=\frac{\mathrm{d}}{\mathrm{d} r} \ln u_{L}(r)
$$


Using above formulae and noting that for the inverse power potentials, i.e. for potentials which fall off asymptotically as const $\cdot r^{-s}$, an analytical solution of the radial Schrödinger equation at $E=0$ had been known, we obtained analytical expressions for the scattering lengths with an exact treatment of short range (SR) parts of the potentials. Then we considered the potentials which have long range (LR) tails vanishing as the inverse fourth power of the distance and we showed that in that case derived formulae had very simple and tractable forms.

In this work we have applied the method described above in order to find the scattering lengths for more general class of LR potentials, so called Lenz potentials of the form:

$$
V_{\text {Lenz }}^{\mp}(r)=\mp \frac{b^{2}}{2 r^{2}\left(x^{\mu}+x^{-\mu}\right)^{2}},
$$

where $x=r / R, r$ is the radial distance from a scattering centre while $R>0, b>0$, $\mu>0$ are parameters. Their focusing properties in classical mechanics as well as in geometrical optics are well known [2]. As was shown by Demkov and Ostrovsky [3], these properties also hold in quantum mechanics. The very important feature of these potentials is that the radial Schrödinger equation at $E=0$ with $V(r)$ given by Eq. (3) has an analytical solution. It means that the corresponding scattering lengths can be calculated analytically, too.

In Sec. 2 we shall derive general formulae for the scattering lengths related to potentials consisting of a short and a long range Lenz part. We shall consider also a particle moving in the "pure" Lenz potential and shall discuss conditions of existence of zero energy bound states. In Sec. 3 we shall discuss an important case when formulae derived in Sec. 2 simplify considerably.

A definition of the scattering length $a_{L}$ is the same as in I. We use again the convention which states that the scattering length has a positive value for the purely repulsive potential. The notation Eq. (I....) refers to equations in the previous paper (I).

\section{Scattering lengths for Lenz potentials}

Let us consider a particle moving in a central potential of the form:

$$
V^{\mp}(r)=\left\{\begin{array}{lll}
U_{\mathrm{S}}^{\mp}(r) & \text { for } & r<d \\
V_{\text {Lenz }}^{\mp}(r) & \text { for } & r \geq d
\end{array}\right.
$$

where $U_{S}^{\mp}(r)$ is the SR part of the interaction potential while $V_{\text {Lenz }}^{\mp}(r)$ is given by Eq. (3). A general solution of the radial Schrödinger equation at $E=0$ and for $r \geq d$

$$
\frac{\mathrm{d}^{2} u \mp}{\mathrm{d} x^{2}}-\frac{L(L+1)}{x^{2}} u_{L}^{\mp} \pm \frac{b^{2}}{x^{2}\left(x^{\mu}+x^{-\mu}\right)^{2}} u_{L}^{\mp}=0
$$

may be written as:

$$
u_{L}^{\mp}(x)=A_{L}^{\mp} x^{L+1} z^{\gamma-1}{ }_{2} F_{1}\left(\xi^{\mp}, \eta^{\mp}, \gamma ; z\right)+B_{L}^{\mp} x^{L+1}{ }_{2} F_{1}\left(\gamma-\xi^{\mp}, \gamma-\eta^{\mp}, 2-\gamma ; z\right),
$$


where

$$
\begin{gathered}
\xi^{\mp}=\frac{2 L+\mu+1-\left(\mu^{2} \pm b^{2}\right)^{1 / 2}}{2 \mu} \\
\eta^{\mp}=\frac{2 L+\mu+1+\left(\mu^{2} \pm b^{2}\right)^{1 / 2}}{2 \mu} \\
\gamma=1+\frac{2 L+1}{2 \mu}
\end{gathered}
$$

and

$$
z=\left(1+x^{2 \mu}\right)^{-1}
$$

It follows from Eqs. (7a)-(7c) that

$$
\xi^{\mp}+\eta^{\mp}+1=2 \gamma
$$

while from Eqs. (7a) and (7b) that $\xi^{+}$and $\eta^{+}$may have real as well as complex (at $\mu^{2}<b^{2}$ ) values. Using Eqs. (1), (6) and Gauss recurrence relations for the hypergeometric function [4] we obtain

$$
a_{L}^{\mp}(r)=r^{2 L+1} \frac{W_{1}\left(\xi^{\mp}, \eta^{\mp}, \gamma ; x\right)+C_{L}^{\mp} W_{2}\left(\xi^{\mp}, \eta^{\mp}, \gamma ; x\right)}{W_{3}\left(\xi^{\mp}, \eta^{\mp}, \gamma ; x\right)-C_{L}^{\mp} W_{4}\left(\xi^{\mp}, \eta^{\mp}, \gamma ; x\right)}, \quad r \geq d
$$

where

$$
\begin{gathered}
W_{1}(\xi, \eta, \gamma ; x)=x^{2 \mu} z^{\gamma} F_{1}(\xi, \eta, \gamma-1 ; z) \\
W_{2}(\xi, \eta, \gamma ; x)=\frac{(\xi-\gamma)(\eta-\gamma)}{(2-\gamma)(\gamma-1)} x^{2 \mu} z_{2} F_{1}(2+\xi-\gamma, 2+\eta-\gamma, 3-\gamma ; z) \\
W_{3}(\xi, \eta, \gamma ; x)=\frac{(\xi-\gamma)(\eta-\gamma)}{\gamma(\gamma-1)} z^{\gamma}{ }_{2} F_{1}(\xi, \eta, \gamma+1 ; z) \\
W_{4}(\xi, \eta, \gamma ; x)={ }_{2} F_{1}(\xi-\gamma, \eta-\gamma, 1-\gamma ; z)
\end{gathered}
$$

and $C_{L}^{\mp}=B_{L}^{\mp} / A_{L}^{\mp}$. Now if $r$ tends to infinity, $a_{L}^{\mp}(r)$ will converge to an exact value of the scaltering length for the potential given by Eq. (4):

$$
a \mp=\lim _{r \rightarrow \infty} a_{L}^{\mp}(r)=R^{2 L+1} \frac{d^{2 L+1} W_{2}\left(\xi^{\mp}, \eta^{\mp}, \gamma ; x_{s}\right)+a_{L_{s}}^{\mp} W_{4}\left(\xi^{\mp}, \eta^{\mp}, \gamma ; x_{s}\right)}{d^{2 L+1} W_{1}\left(\xi^{\mp}, \eta^{\mp}, \gamma ; x_{s}\right)-a_{L_{s}} W_{3}\left(\xi^{\mp}, \eta^{\mp}, \gamma ; x_{s}\right)},
$$

where $x_{s}=d / R$ and $a \dot{\bar{L}}_{s} \equiv a_{L}^{\mp}(d)$ is the scattering length related to the SR part of the potential. As follows from the direct evaluation of Eq. (11), $a_{L}^{\mp}$ exists only when a relation $2 L+1<2 \mu$, i.e. $\gamma<2$ holds (compare Eq. (I.2)). From the properties of the hypergeometric function one finds that in the limit $R \rightarrow 0$ and $b^{2} R^{2 \mu} \rightarrow \beta^{2}, a_{L}^{\mp}$ tends to the scattering lengths given by Eqs. (I.13) and (I.14).

In many cases it is sufficient to know the scattering lengths to first order in $b^{2} R^{2 \mu}$ and $b^{2} R^{4 \mu}$. At that time Eq. (11) can be expressed approximately as:

$$
a_{L}^{\mp}=a_{L s}^{\mp} \mp b^{2} R^{2 \mu} \theta_{L}^{(1) \mp} \pm 2 b^{2} R^{4 \mu} \theta_{L}^{(2) \mp}
$$


where

$$
\theta_{L}^{(k) \mp}=\frac{1}{2 k \mu(2 L+1) d^{2 k \mu\left(1+\nu_{k}\right)}}\left(\frac{d^{2(2 L+1)}}{1-\nu_{k}}-2 a \mp_{s} d^{2 L+1}+\frac{\left(a_{L_{s}}\right)^{2}}{1+\nu_{k}}\right)
$$

and

$$
\nu_{k}=\frac{2 L+1}{2 k \mu}
$$

Eq. (12) can be also obtained in an alternative way. Indeed, it results from Eq.(3) that to first order in $b^{2} R^{2 \mu}$ and $b^{2} R^{4 \mu}$ we have

$$
V_{\text {Lenz }}^{\mp}(r) \approx \mp \frac{b^{2} R^{2 \mu}}{2 r^{2 \mu+2}} \pm \frac{2 b^{2} R^{4 \mu}}{2 r^{4 \mu+2}}
$$

and now employing Eqs. (I.20) and (I.21) we again obtain Eq. (12).

Finally let us examine the scattering length on the "pure" Lenz potential, i.e. on the potential (4) which does not contain the SR part. We have

$$
\lim _{d \rightarrow 0} a_{L}^{\mp}=R^{2 L+1} \frac{\Gamma(2-\gamma) \Gamma\left(\xi^{\mp}\right) \Gamma\left(\eta^{\mp}\right)}{\Gamma(\gamma) \Gamma\left(\gamma-\xi^{\mp}\right) \Gamma\left(\gamma-\eta^{\mp}\right)} .
$$

It should be emphasized that, in contrast with the case of the scattering on the purely attractive inverse power potential, now $\lim _{d \rightarrow 0} a_{L}^{-}$is well defined.

It is well known that bound states with angular momentum $L$ and energy $E=0$ appear if $a_{L}=\mp \infty$. As follows from Eq. (16), in the case of the "pure" attractive Lenz potential it occurs when $\Gamma\left(\xi^{-}\right)$has the poles, i.e. when $\xi^{-}=-n_{r}$, where $n_{r}=0,1,2, \ldots$ can be identified with the radial quantum number. It is equivalent to the requirement:

$$
b^{2}=\left[\left(2 n_{r}+1\right) \mu+(2 L+1)\right]^{2}-\mu^{2},
$$

which was first found by Demkov and Ostrovsky (see Eq. (25) in [3]).

$$
\text { 3. } \gamma=3 / 2 \text { case }
$$

In the particular case, when $\gamma=3 / 2$, i.e. when $2 L+1=\mu$, Eq. (11) can be simplified considerably. We have

$$
\xi^{\mp}=1-\frac{\lambda^{\mp}}{2} \text { and } \eta^{\mp}=1+\frac{\lambda^{\mp}}{2},
$$

where

$$
\lambda^{\mp}=\left(1 \pm \frac{b^{2}}{\mu^{2}}\right)^{1 / 2}
$$

so the hypergeometric functions in Eqs. (10a)-(10d) can be expressed through elementary functions [4] and

$$
a_{L}^{-}=\lambda^{-} R^{\mu} \frac{\left(R^{2 \mu}+a_{L s}^{-} d^{\mu}\right)-\lambda^{-} R^{\mu}\left(d^{\mu}-a_{L s}^{-}\right) \tan \Omega^{-}}{\left(R^{2 \mu}+a_{L s}^{-} d^{\mu}\right) \tan \Omega^{-}+\lambda^{-} R^{\mu}\left(d^{\mu}-a_{L s}^{-}\right)}
$$




$$
\begin{gathered}
a_{L}^{+}=\lambda^{+} R^{\mu} \frac{\left(R^{2 \mu}+a_{L s}^{+} d^{\mu}\right)-\lambda^{+} R^{\mu}\left(d^{\mu}-a_{L s}^{+}\right) \tan \Omega^{+}}{\left(R^{2 \mu}+a_{L s}^{+} d^{\mu}\right) \tan \Omega^{+}+\lambda^{+} R^{\mu}\left(d^{\mu}-a_{L s}^{+}\right)}, \quad b<\mu \\
a_{L}^{+}=\left|\lambda^{+}\right| R^{\mu} \frac{\left(R^{2 \mu}+a_{L s}^{+} d^{\mu}\right)+\left|\lambda^{+}\right| R^{\mu}\left(d^{\mu}-a_{L s}^{+}\right) \tanh \Omega^{+}}{\left(R^{2 \mu}+a_{L s}^{+} d^{\mu}\right) \tanh \Omega^{+}+\left|\lambda^{+}\right| R^{\mu}\left(d^{\mu}-a_{L s}^{+}\right)}, \quad b>\mu
\end{gathered}
$$

where

$$
\Omega^{\mp}=\left|\lambda^{\mp}\right| \arctan \left(\frac{R}{d}\right)^{\mu} \text {. }
$$

In the limit $R \rightarrow 0$ and $b^{2} R^{2 \mu} \rightarrow \beta^{2}$ right hand sides of Eqs. (20a) and (20c) tend to the scattering lengths given by Eqs. (I.15a) and (I.15b).

Equation (20a) provides a very simple and very accurate method of calculation of $a_{0}$ for the potentials vanishing asymptotically as Buckingham polarization potential:

$$
V(r) \approx-\frac{\alpha}{2\left(r^{2}+R^{2}\right)^{2}}, \quad r \geq d
$$

which is a model potential describing the interaction between a neutral polarizable target and a charged projectile. In this case one should put in Eqs. (19), (20a) and (21) $L=0, \mu=1$ and $b^{2} R^{2}=\alpha$, where $\alpha$ is the electric dipole polarizability of the target.

Finally, let us note that for $\gamma=3 / 2$, Eq. (16) reduces to

$$
\begin{aligned}
& \lim _{d \rightarrow 0} a_{L}^{-}=\lambda^{-} R^{\mu} \cot \left(\pi \lambda^{-} / 2\right), \\
& \lim _{d \rightarrow 0} a_{L}^{+}=\lambda^{+} R^{\mu} \cot \left(\pi \lambda^{+} / 2\right) \quad \text { for } \quad b<\mu, \\
& \lim _{d \rightarrow 0} a_{L}^{+}=\left|\lambda^{+}\right| R^{\mu} \operatorname{coth}\left(\pi\left|\lambda^{+}\right| / 2\right) \quad \text { for } \quad b>\mu .
\end{aligned}
$$

\section{Conclusions}

We have applied the method described in the previous paper in order to calculate the scattering lengths for potentials vanishing asymptotically as Lenz potentials. It was shown that derived expressions have especially simple form for potentials which fall off asymptotically as Buckingham polarization potential. We have also considered conditions of existence of zero energy bound states in the "pure" Lenz potentials. The obtained result agrees with that found previously by Demkov and Ostrovsky.

\section{Erratum}

There are some misprints in our previous paper I. Equation (I.20) should read

$$
a_{L}^{\mp}=a_{L s}^{\mp} \mp \beta^{2} \theta_{L}^{\mp}
$$


and Eq. (I.21) should read

$$
\theta_{L}=\frac{p}{2 L+1}\left(\frac{d^{2(2 L+1)}}{1-\nu}-2 a_{E_{s}} d^{2 L+1}+\frac{\left(a_{E_{s}}\right)^{2}}{1+\nu}\right) \frac{1}{d(\nu+1) / p} .
$$

\section{References}

[1] R. Szmytkowski, Acta Phys. Pol. A78, 517 (1990).

[2] R.K. Luneburg, Mathematical Theory of Optics, University of California Press, Berkeley and Los Angeles 1964.

[3] Yu.N. Demkov, V.N. Ostrovsky, Zh. Eksp. Teor. Fiz. 60, 2012 (1971).

[4] I.S. Gradshteyn, I.M. Ryzhik, Tables of Integrals, Sums, Series and Products, 5th ed. (in Russian), Nauka, Moscow 1971, Chap. 9.1. 